\title{
Identificación de Escherichia coli enteropatógena en niños con síndrome diarreico agudo del Estado Sucre, Venezuela
}

\author{
Elvia Michelli ${ }^{1,2}$, Adriana Millán ${ }^{1}$, Hectorina Rodulfo', Mirian Michelli', \\ Jesús Luiggi ${ }^{1}$, Numirin Carreño ${ }^{1}$, Marcos de Donato ${ }^{1}$
}

\author{
1 Laboratorio de Genética Molecular, Instituto de Investigaciones en Biomedicina y Ciencias Aplicadas "Dra. \\ Susan Tai", Universidad de Oriente, Cumaná, Sucre, Venezuela \\ 2 Laboratorio de Microbiología Clínica, Departamento de Bioanálisis, Universidad de Oriente, Cumaná, Sucre, \\ Venezuela
}

Introducción. Escherichia coli es uno de los principales agentes causales del síndrome diarreico agudo. Objetivo. Identificar grupos clonales de E. coli enteropatógena en 485 casos de diarrea aguda en niños entre 0 y 10 años de edad atendidos en centros de salud de los municipios de Arismendi, Benítez y Sucre del estado Sucre, Venezuela, entre marzo y diciembre de 2011.

Materiales y métodos. Previo consentimiento informado, se recolectaron muestras fecales y se identificó E. colimediante coprocultivo estándar y serología con antisueros polivalentes y monovalentes. Se aisló el ADN y se amplificaron los genes eae (intimina) y bfpA (bundlina) mediante dos pruebas de reacción en cadena de la polimerasa $(\mathrm{PCR})$ múltiples.

Resultados. En 39,6 \% de los coprocultivos se determinó la presencia de infección bacteriana. La prevalencia de E. coli fue de 54,7 \%; 82,9 \% de estas cepas fue positivo por serología para los serogrupos y el serotipo evaluados, principalmente en niños entre los 0 y los 2 años (37,9\%). El 48,6 \% de las cepas de E. coli amplificaron para el gen eae y, de estas, 58,8 \% se clasificó como cepas de E. coli enteropatógena típica (eae+ y bfp+). EI ECEP II fue el serogrupo más frecuente $(38,7 \%)$, con predominio de bacterias E. coli enteropatógenas típicas (60\%). El alelo $\beta$ de la intimina fue el más identificado $(74,5 \%)$ en las cepas positivas para el gen eae. Solo se identificaron cuatro cepas con el serotipo O157:H7 utilizando antisueros, las cuales no amplificaron mediante PCR para los genes eae y $b f p A$.

Conclusiones. Este estudio demostró la importancia de aplicar pruebas moleculares en la identificación de las cepas de E. coli causantes de diarrea de diversa gravedad.

Palabras clave: Escherichia coli, Escherichia coli enteropatógena, enfermedades gastrointestinales, diarrea, reacción en cadena de la polimerasa.

doi: http://dx.doi.org/10.7705/biomedica.v36i0.2928

\section{Identification of enteropathogenic Escherichia coli in children with acute diarrheic syndrome from Sucre State, Venezuela}

Introduction: Diarrheagenic Escherichia coli is an important causative agent of acute diarrheic syndrome. Objective: To identify clonal groups of enteropathogenic E. coli (EPEC), in 485 children with acute diarrhea aged 0 to 10 years attending health care centers in Arismendi, Benítez and Sucre municipalities, Sucre state, Venezuela, from March to December, 2011.

Materials and methods: After obtaining the informed consent, stool samples were collected. Escherichia coli was identified using standard coproculture methods and serology with polyvalent and monovalent antisera. DNA was isolated, and eae (intimin) and bfpA (bundlin) genes were amplified through two multiplex polymerase chain reactions (PCR).

Results: The presence of bacterial infection was determined in $39.6 \%$ of coprocultures. The prevalence of $E$. coli was $54.7 \%$; $82.9 \%$ of these isolates were positive by serology for the evaluated serogroups and serotypes, which were mostly identified in children between 0 and 2 years (37.9\%); $48.6 \%$ of $E$. coli strains amplified the eae gene; of these, $58.8 \%$ were classified as typical EPEC (eae+ y bfp+). EPEC II was the most common serogroup (38.7\%), with predominance of typical EPEC

\section{Contribución de los autores:}

Elvia Michelli, Hectorina Rodulfo y Marcos De Donato: concepción y diseño del estudio

Adriana Millán, Mirian Michelli, Jesús Luiggi y Numirin Carreño: recolección y procesamiento de muestras

Elvia Michelli: redacción del manuscrito

Todos los autores participaron en el análisis y la interpretación de los datos. 
(60\%). In positive strains for eae gene, the $\beta$ intimin allele was the most frequently identified $(74.5 \%)$. Only four strains with O157:H7 serotype were identified, which showed no PCR amplification of the eae and $b f p A$ genes.

Conclusion: This study showed the importance of molecular tests to identify diarrheagenic $E$. coli strains causing clinical conditions of varying severity.

Key words: Escherichia coli, enterophatogenic Escherichia coli, gastrointestinal diseases, diarrhea, polymerase chain reaction.

doi: http://dx.doi.org/10.7705/biomedica.v36i0.2928

El síndrome diarreico agudo representa un problema de salud pública mundial, aunque con variaciones regionales en su incidencia, y en la diversidad y frecuencia de los agentes etiológicos involucrados.

Entre los microorganismos causantes de diarrea aguda se encuentran las cepas de Escherichia coli, las cuales se clasifican en los siguientes seis patotipos según sus características clínicas y epidemiológicas, y los factores específicos que determinan su virulencia: $E$. coli enteropatógena (ECEP), E. coli enterohemorrágica (ECEH), E. coli enterotoxigénica (ECET), E. coli enteroinvasiva (ECEI), E. coli enteroagregativa (ECEAgg) y E. coli de adherencia difusa (ECAD) (1-3).

Las cepas ECEP causan diarreas acuosas; la adherencia a los enterocitos es un evento clave en su patogenia, la cual tiene dos fases. La primera fase involucra la expresión de los genes bfp (codificados en el plásmido denominado factor de adherencia a EPEC, pEAF), los cuales codifican las fimbrias formadoras de penachos (bundle forming pili, BFP), las que, a su vez, median la formación de microcolonias, originando un fenotipo llamado de adherencia localizada $(4,5)$. Según se dé la presencia del plásmido EAF o no, las ECEP se clasifican como ECEP típicas, que contienen dicho plásmido, y ECEP atípicas, las cuales no lo poseen. Las cepas ECEP atípicas presentan una adherencia semejante a la localizada, la difusa o la agregativa (5-7). La segunda fase de la adherencia está definida por la expresión del gen eae codificado en la isla de patogenia denominada locus de borrado del enterocito (Locus of Enterocyte Effacement, LEE), que codifica la proteína intimina.

\footnotetext{
Correspondencia:

Elvia Michelli, Laboratorio de Genética Molecular, Instituto de Investigaciones en Biomedicina y Ciencias Aplicadas "Dra. Susan Tai", Universidad de Oriente, Avenida Universidad, Cerro del Medio, Cumaná, Sucre, Venezuela Teléfonos: (58-412) 6993098 y (293) 4515684 melvia@udo.edu.ve y elviamichell@yahoo.com

Recibido: 13/07/15; aceptado: 19/03/16
}

Esta proteína media la formación del fenotipo de adherencia y borrado (attaching and effacing, A/E), caracterizado por la adherencia íntima de la bacteria al enterocito con la subsiguiente destrucción de las microvellosidades y el rearreglo del citoesqueleto celular, lo cual resulta en la formación de una estructura en forma de pedestal en el sitio de unión de la bacteria y el enterocito $(6,8,9)$.

En varios estudios a nivel mundial se ha demostrado que los patotipos de E. coli causante de diarrea usualmente están involucrados en la etiología de la diarrea en niños $(3,10)$, con una elevada frecuencia de ECEP típicas y atípicas como agente patógeno único o asociado a otros agentes microbianos (1116). Asimismo, diversos estudios en Venezuela coinciden en señalar el importante papel de estas cepas en el síndrome diarreico agudo infantil, como se demostró en una investigación de 754 niños de Caracas, en la cual se determinó la significación epidemiológica de los aislamientos de $E$. coli con fenotipo de adherencia localizada en cultivos celulares (17). En una investigación de 200 niños con síndrome diarreico agudo en Cumaná, estado Sucre, se obtuvieron 169 aislamientos de E. coli, en los cuales las cepas ECEP fueron las más frecuentemente identificadas (10,7 \%: 1,2 \%, ECEP típicas y 9,5\%, ECEP atípicas) (18). En otro estudio en Cumaná, se sugirió que había circulación de cepas de E. coli causantes de diarrea y productoras de Stx (Shiga-like toxins) diferentes al serotipo 0157:H7, y se señaló su participación en la etiología del síndrome diarreico agudo en niños menores de tres años (19).

La diversidad genética de las cepas de $E$. coli causantes de diarrea ha puesto de relieve la utilidad de las herramientas moleculares en los estudios epidemiológicos orientados a la identificación y clasificación de los patotipos mediante la detección de genes cromosómicos o plasmídicos responsables de su patogenia, los cuales están ausentes en las cepas comensales de E. coli $(9,20)$. Dado que el síndrome diarreico agudo es una causa importante de morbimortalidad en niños y que las cepas de 
$E$. coli causantes de diarrea se encuentran entre sus principales agentes etiológicos, en este trabajo se identificaron los grupos clonales de ECEP mediante técnicas moleculares en niños con síndrome diarreico agudo atendidos en centros asistenciales del estado Sucre, con el fin de caracterizar la epidemiología de las cepas circulantes y, así, contribuir en la adopción de las medidas de control pertinentes.

\section{Materiales y métodos}

\section{Tipo de estudio}

Se hizo un estudio descriptivo de corte transversal.

\section{Criterios de inclusión y de exclusión}

Los pacientes incluidos en el estudio presentaban síndrome diarreico agudo con un tiempo de evolución no mayor de 72 horas en el momento de su evaluación clínica; se excluyeron del estudio todos los niños que habían recibido tratamiento antimicrobiano durante el mes anterior a la toma de muestras.

\section{Muestras}

Entre los meses de marzo y diciembre de 2011 se recolectaron 485 muestras fecales por emisión espontánea de niños entre los 0 y 10 años de edad, que luego se guardaron en envases plásticos estériles; 84 de los niños fueron atendidos en el Ambulatorio de Caituco y, 74, en el de Llanada de Cangua, en los municipios rurales de Benítez y Arismendi, respectivamente. Los otros 327 niños asistieron al Laboratorio Clínico Comunitario y a los Ambulatorios de La Llanada y Brasil, parroquia Altagracia, al Ambulatorio "Dr. Ramón Martínez" de Las Palomas, parroquia Ayacucho, y al Hospital Ambulatorio "Salvador Allende", parroquia Valentín Valiente, todos ubicados en el municipio de Sucre del estado Sucre, Venezuela.

\section{Diagnóstico microbiológico}

En las dos horas siguientes a su recolección, una porción de las muestras fecales se emulsionó en infusión de cerebro y corazón, luego se inoculó por extensión y agotamiento en placas con los siguientes medios de cultivo selectivos diferenciales McConkey: Salmonella-Shigella, eosina de azul de metileno y agar de xilosa, lisina y desoxicolato de sodio. Posteriormente, las placas se incubaron en aerobiosis a $35^{\circ} \mathrm{C}$ durante 18 a 24 horas (21).

Las colonias con características fenotípicas sugestivas de $E$. coli se identificaron mediante técnicas bioquímicas, siguiendo los protocolos estándar
$(21,22)$. A todas las cepas confirmadas como $E$. coli se les hizo la prueba de sorbitol. En las pruebas bioquímicas se utilizaron las cepas certificadas de E. coli (ATCC 25922) y Pseudomonas aeruginosa (ATCC 27853) como controles positivo y negativo, respectivamente, provenientes del Centro Venezolano de Colecciones Microbiológicas (CVCM).

\section{Caracterización serológica de las cepas de Escherichia coli}

Los serogrupos de las cepas de $E$. coli se determinaron mediante la técnica de aglutinación en láminas a partir de un cultivo puro en agar nutritivo y con antisueros polivalentes, con los siguientes resultados: ECEP: I (O26, O55, 0111 y O45), II (O86, O119, O127 y O88), III (O125, O126 y 0128) y IV (O114, O142 y O158); ECET: I (O6, O25, O27, O78 y O168), II (O26, O63, O155, O166 y O148) y III (O8, O15, O115, O159 y O169); y ECEI: I (O112ac, O152 y O164), II (O29, O143 y O144) y III (O28, O124, O136 y O167). Con el antisuero monovalente para ECEH se identificó el serotipo O157:H7. La identificación se llevó a cabo según el procedimiento sugerido por la Fundación Venezolana para el Estudio de la Salud Infantil (FUVESIN) (23). Se utilizaron como controles positivos las cepas de E. coli identificadas como CVCM 514 (O55:K59), CVCM 511 (O86:H34), CVCM 516 (O125:K70:H19), CVCM 1014 (O114:K90:H32) y CVCM 442 (O157:H7), y la cepa de $P$. aeruginosa ATCC 27853 se usó como control negativo.

\section{Diagnóstico molecular de ECEP y ECEH}

EI ADN se aisló a partir de cepas de E. coli identificadas bioquímicamente y cultivadas en caldo Luria Bertani, las cuales se incubaron a $37^{\circ} \mathrm{C}$ durante toda la noche, utilizando el estuche comercial Wizard Genomic DNA Purification $\mathrm{Kit}^{\mathrm{TM}}$ (Promega) y siguiendo las especificaciones del fabricante. Se hizo una reacción en cadena de la polimerasa (PCR) múltiple con los oligos AE11/ eaek4 (amplicón de 526 pb del gen eae), que codifica para la proteína intimina, y BFP3/BFP2 (amplicón de 209 pb del gen bfpA), que codifica para la bundlina de la fimbria BFP, siguiendo los protocolos descritos previamente $(24,25)$.

En la identificación de las formas alélicas a, $\beta$ y $\gamma$ del gen eae, se utilizó otra PCR múltiple siguiendo el procedimiento descrito por Reid, et al. (26), en el cual se utilizaron simultáneamente el par de oligonucleótidos eae P1/P2, que amplifican un fragmento de 917 pb común para las cepas de ECEP y ECEH, y los pares de oligonucleótidos 
P1/Ecoeae $\alpha$ (amplicón 1.648 pb), P1/Ecoeae $\beta$ (amplicón 1.926 pb) y P1/Ecoeaey (amplicón 1.770 pb), cuyos amplicones son específicos de las cepas ECEP 1, ECEP 2 y ECEH 1, respectivamente (26).

En las PCR se utilizaron como controles positivos las cepas de E. coli ATCC 25922, CVCM 442 (O157:H7), CVCM 511 (O86:H34), CVCM 988 (O78:H12) y CVCM 514 (O55:K59), y la ATCC 27853 de $P$. aeruginosa como control negativo.

Todas las reacciones de amplificación se hicieron en un equipo $\mathrm{ABI} 2400$ (Applied Biosystems) y los productos amplificados se visualizaron por electroforesis en geles de agarosa al $2 \%$ teñidos con bromuro de etidio $(0,05 \%)$ bajo luz ultravioleta.

\section{Análisis de los resultados}

Las cepas ECEP se clasificaron como típicas cuando amplificaron los genes eae y $b f p A$ (eae+ y bfp+), mientras que las ECEP atípicas se definieron como eae+ y bfp- (8). El análisis de los datos clínicos y epidemiológicos se hizo mediante la prueba de ji al cuadrado con el programa estadístico SPSS ${ }^{\circledR}$ (versión 18). Todas las pruebas se hicieron con $95 \%$ de confiabilidad.

\section{Consideraciones éticas}

A los padres o representantes de los niños se les solicitó firmar un consentimiento después de informarles la naturaleza y la importancia de la investigación, así como sobre los posibles riesgos. Se les aclaró que el estudio se ajustaría a los principios establecidos en la Declaración de Helsinki (27). En el manejo de los pacientes y de su información se siguieron las normas de bioética y bioseguridad establecidas por la Comisión de Bioética y Bioseguridad del Instituto de Investigaciones en Biomedicina y Ciencias Aplicadas "Dra. Susan Tai".

\section{Resultados}

A partir de los coprocultivos de los 485 pacientes incluidos en el estudio, se determinó la presencia de infecciones bacterianas en $39,6 \%$ de ellos (192/485), sobre todo en los pacientes de 0 a 4 años de edad ( $51 \%$ ) y en los provenientes del municipio de Sucre (92,2\%) (cuadro 1). En 54,69 \% (105/192) de las muestras positivas se identificó $E$. coli. En los pacientes portadores de E. coli se reportó más de uno de los signos y síntomas clínicos asociados al síndrome diarreico agudo.

Cuadro 1. Distribución de los niños con coprocultivos positivos e identificación de Escherichia coli según su procedencia, grupos de edad (de dos años cada uno), sexo y características clínicas asociadas al síndrome diarreico agudo

\begin{tabular}{|c|c|c|c|c|c|c|c|c|}
\hline \multirow[t]{2}{*}{ Parámetros } & \multicolumn{2}{|c|}{ Individuos con SDA } & \multicolumn{3}{|c|}{ Coprocultivos positivos } & \multicolumn{3}{|c|}{ E. coli positiva } \\
\hline & $\mathbf{n}$ & $\%$ & $\mathbf{n}$ & $\%$ & $\mathbf{p}$ & $\mathbf{n}$ & $\%$ & $\mathbf{p}$ \\
\hline Procedencia & & & & & $<0,0001^{*}$ & & & $<0,0001^{*}$ \\
\hline Rural ${ }^{¥}$ & $158 / 485$ & 32,58 & $15 / 158$ & 9,49 & & $7 / 15$ & 46,67 & \\
\hline Urbana $^{£}$ & $327 / 485$ & 67,43 & $177 / 327$ & 54,13 & & $98 / 177$ & 55,37 & \\
\hline Sexo & & & & & 0,0397 & & & 1,9975 \\
\hline Femenino & 249/485 & 51,34 & $97 / 249$ & 38,96 & & $47 / 97$ & 48,45 & \\
\hline Masculino & $236 / 485$ & 48,66 & $95 / 236$ & 40,25 & & $58 / 95$ & 61,05 & \\
\hline Grupos de edad (años) & & & & & $0,0070^{*}$ & & & 0,6013 \\
\hline 0 a 2 & $177 / 485$ & 36,50 & $65 / 177$ & 36,72 & & $41 / 65$ & 63,08 & \\
\hline 3 a 4 & $82 / 485$ & 16,90 & $33 / 82$ & 40,24 & & $16 / 33$ & 48,48 & \\
\hline 5 a 6 & $77 / 485$ & 15,90 & $27 / 77$ & 35,06 & & $13 / 27$ & 48,15 & \\
\hline 7 a 8 & $57 / 485$ & 11,80 & $16 / 57$ & 28,07 & & $11 / 16$ & 68,75 & \\
\hline 9 a 10 & $92 / 485$ & 19,00 & $51 / 92$ & 55,43 & & $24 / 51$ & 47,06 & \\
\hline \multicolumn{9}{|l|}{ Características clínicas } \\
\hline Moco§ & $105 / 485$ & 21,65 & $47 / 105$ & 44,76 & 0,8976 & $24 / 47$ & 51,06 & $0,0045^{*}$ \\
\hline Sangre§ & $1 / 485$ & 0,21 & $0 / 1$ & - & - & - & - & - \\
\hline Moco y sangre $\$$ & $14 / 485$ & 2,89 & $5 / 14$ & 35,71 & 0,1339 & $2 / 5$ & 40,00 & 1,2466 \\
\hline DA & $76 / 485$ & 15,67 & $30 / 76$ & 39,47 & 0,1816 & $17 / 30$ & 56,67 & 1,1050 \\
\hline $\mathrm{F}$ & $97 / 485$ & 20,00 & $28 / 97$ & 28,87 & 0,1108 & $13 / 28$ & 46,43 & 0,1611 \\
\hline $\mathrm{V} / \mathrm{N}$ & $47 / 485$ & 9,69 & $16 / 47$ & 34,04 & $0,0117^{*}$ & $8 / 16$ & 50,00 & $0,0117^{*}$ \\
\hline $\mathrm{DA} / \mathrm{F}$ & $31 / 485$ & 6,39 & $10 / 31$ & 32,26 & 0,1595 & $7 / 10$ & 70,00 & $0,0006^{*}$ \\
\hline $\mathrm{DA} / \mathrm{V} / \mathrm{N}$ & $11 / 485$ & 2,27 & $8 / 11$ & 72,73 & 0,0735 & $4 / 8$ & 50,00 & $0,0071^{*}$ \\
\hline $\mathrm{DA} / \mathrm{F} / \mathrm{V} / \mathrm{N}$ & $17 / 485$ & 3,51 & $8 / 17$ & 47,06 & 0,9015 & $6 / 8$ & 75,00 & $0,0032^{*}$ \\
\hline $\mathrm{F} / \mathrm{V} / \mathrm{N}$ & $13 / 485$ & 2,68 & $8 / 13$ & 61,54 & 0,3160 & $6 / 8$ & 75,00 & 0,8933 \\
\hline
\end{tabular}

SDA: síndrome diarreico agudo; Rural*: municipios de Arismendi y Benítez; Urbana ₹: municipio de Sucre; §: características macroscópicas de las muestras fecales; DA: dolor abdominal; F: fiebre; V: vómitos; N: náuseas; *: prueba de ji al cuadrado estadísticamente significativa 
En el cuadro 2 se presenta la identificación serológica de las 105 cepas de E. coli, de las cuales $82,9 \%$ (87/105) fue positivo para alguno de los serogrupos y serotipos evaluados, mientras que $17,1 \%(18 / 105)$ de ellas no aglutinó. El serogrupo ECEP II fue el más frecuente, con 38,9\% (38/105), seguido del ECEP III, con 18,9 \% (19/105); en una cepa se identificó el ECEI y en cuatro cepas, el ECEH. La distribución de los serogrupos identificados según la procedencia de los pacientes, evidenció su predominio en las muestras fecales recolectadas en el municipio urbano de Sucre; los más frecuentes fueron los serogrupos ECEP II, con $38,8 \%$ (38/98), y ECEP III, con $19,4 \%$ (19/98); las cuatro cepas de ECEH identificadas también se encontraron en pacientes del municipio de Sucre, mientras que la única cepa ECEI se identificó en un paciente procedente de una zona rural (Llanada de Cangua). Los serogrupos de $E$. coli se distribuyeron de forma similar en niños. Los serogrupos de E. coli identificados se presentaron con mayor frecuencia en los pacientes entre $0 \mathrm{y}$ 2 años de edad, con 37,9 \% (33/87), sobre todo el ECEP II, con 57,6\% (19/33), y el ECEP III, con $21,2 \%(7 / 33)$ (cuadro 2).

En la figura $1 \mathrm{~A}$ se presentan parcialmente los resultados obtenidos a partir de la amplificación mediante PCR múltiple de los genes eae (intimina) y bfpA (bundlina) en las cepas de E. coli, y se observa que hubo amplificación para el gen eae solo y para ambos genes; sin embargo, el gen bfp siempre amplificó junto con intimina en las muestras evaluadas. En la figura 1B se observan ejemplos de la amplificación de las formas alélicas $\alpha, \beta$ y $\gamma$ del gen eae.

En el cuadro 3 se presentan en detalle los resultados de las pruebas de PCR según la clasificación serológica de las 105 cepas de E. coli (17 cepas ECEP I, 38 ECEP II, 19 ECEP III, 8 ECEP IV, 4 ECEH, 1 ECEI y 18 cepas que no aglutinaron); se resalta el hecho de que en $48,6 \%(51 / 105)$ de los

Cuadro 2. Distribución de los serotipos de Escherichia coli identificados en los niños estudiados según su procedencia, grupos de edad (de dos años cada uno), sexo y características clínicas asociadas al síndrome diarreico agudo

\begin{tabular}{|c|c|c|c|c|c|c|c|c|c|c|c|c|c|c|}
\hline \multirow[t]{3}{*}{ Parámetros } & \multicolumn{14}{|c|}{ Serotipificación de Esherichia coli } \\
\hline & \multicolumn{2}{|c|}{ ECEP 1} & \multicolumn{2}{|c|}{ ECEP 2} & \multicolumn{2}{|c|}{ ECEP 3} & \multicolumn{2}{|c|}{ ECEP 4} & \multicolumn{2}{|c|}{ ECEH } & \multicolumn{2}{|c|}{ ECEI 1} & \multicolumn{2}{|c|}{ NA } \\
\hline & $\mathbf{n}$ & $\%$ & $\mathbf{n}$ & $\%$ & $\mathbf{n}$ & $\%$ & $\mathbf{n}$ & $\%$ & $\mathbf{n}$ & $\%$ & $\mathbf{n}$ & $\%$ & $\mathbf{n}$ & $\%$ \\
\hline \multicolumn{15}{|l|}{ Procedencia } \\
\hline Rural ${ }^{¥}$ & 2 & 11,76 & - & - & - & - & 2 & 25,00 & - & - & 1 & 100,0 & 2 & 11,11 \\
\hline Urbana ${ }^{£}$ & 15 & 88,24 & 38 & 100,0 & 19 & 100,0 & 6 & 75,00 & 4 & 100,0 & - & - & 16 & 88,89 \\
\hline Total & 17 & 100,0 & 38 & 100,0 & 19 & 100,0 & 8 & 100,0 & 4 & 100,0 & 1 & 100,0 & 18 & 100,0 \\
\hline \multicolumn{15}{|l|}{ Sexo } \\
\hline Femenino & 8 & 47,06 & 20 & 52,63 & 6 & 31,58 & 4 & 50,00 & 1 & 25,00 & 1 & 100,0 & 7 & 38,89 \\
\hline Masculino & 9 & 52,94 & 18 & 47,37 & 13 & 68,42 & 4 & 50,00 & 3 & 75,00 & - & - & 11 & 61,11 \\
\hline Total & 17 & 100,0 & 38 & 100,0 & 19 & 100,0 & 8 & 100,0 & 4 & 100,0 & 1 & 100,0 & 18 & 100,0 \\
\hline \multicolumn{15}{|c|}{ Grupos de edad (años) } \\
\hline 0 a 2 & 4 & 23,53 & 19 & 50,00 & 7 & 36,84 & 2 & 25,00 & 1 & 25,00 & - & - & 8 & 44,44 \\
\hline 3 a 4 & 4 & 23,53 & 3 & 7,89 & 4 & 21,05 & - & - & 1 & 25,00 & - & - & 4 & 22,22 \\
\hline 5 a 6 & 1 & 5,88 & 2 & 5,26 & 3 & 15,79 & 5 & 62,50 & 1 & 25,00 & 1 & 100,0 & - & - \\
\hline 7 a 8 & 2 & 11,76 & 5 & 13,16 & 2 & 10,53 & - & - & - & - & - & - & 2 & 11,11 \\
\hline 9 a 10 & 6 & 35,29 & 9 & 23,68 & 3 & 15,79 & 1 & 12,50 & 1 & 25,00 & - & - & 4 & 22,22 \\
\hline Total & 17 & 100,0 & 38 & 100,0 & 19 & 100,0 & 8 & 100,0 & 4 & 100,0 & 1 & 100,0 & 18 & 100,0 \\
\hline \multicolumn{15}{|c|}{ Características clínicas } \\
\hline Solo diarrea & 2 & 11,76 & 7 & 18,42 & 6 & 31,58 & 2 & 25,00 & 2 & 50,00 & 1 & 100,0 & 3 & 16,67 \\
\hline Moco $\$$ & 3 & 17,65 & 9 & 23,68 & 3 & 15,79 & 3 & 37,50 & - & - & - & - & 5 & 27,28 \\
\hline Moco y sangre $\$$ & 1 & 5,88 & - & - & - & - & - & - & - & - & - & - & 1 & 5,56 \\
\hline DA & 2 & 11,76 & 6 & 15,79 & 4 & 21,05 & 1 & 12,50 & 1 & 25,00 & - & - & 3 & 16,67 \\
\hline $\mathrm{F}$ & 2 & 11,76 & 6 & 15,79 & - & - & - & - & - & - & - & - & 3 & 16,67 \\
\hline $\mathrm{V} / \mathrm{N}$ & 4 & 23,53 & 1 & 2,63 & 4 & 21,05 & - & - & - & - & - & - & - & - \\
\hline $\mathrm{DA} / \mathrm{F}$ & 3 & 17,65 & 1 & 2,63 & - & - & 1 & 12,50 & 1 & 25,00 & - & - & - & - \\
\hline $\mathrm{DA} / \mathrm{V} / \mathrm{N}$ & - & - & 1 & 2,63 & 1 & 5,26 & - & - & - & - & - & - & 1 & 5,56 \\
\hline $\mathrm{DA} / \mathrm{F} / \mathrm{V} / \mathrm{N}$ & - & - & 3 & 7,89 & 1 & 5,26 & 1 & 12,50 & - & - & - & - & 1 & 5,56 \\
\hline $\mathrm{F} / \mathrm{V} / \mathrm{N}$ & - & - & 4 & 10,53 & - & - & - & - & - & - & - & - & 1 & 5,56 \\
\hline Total & 17 & 100,0 & 38 & 100,0 & 19 & 100,0 & 8 & 100,0 & 4 & 100,0 & 1 & 100,0 & 18 & 100,0 \\
\hline
\end{tabular}

SDA: síndrome diarreico agudo; Rural¥: municipios de Arismendi y Benítez; Urbano ₹: municipio de Sucre; §: características macroscópicas de las muestras fecales; NA: no aglutinante; DA: dolor abdominal; F: fiebre; V: vómitos; N: náuseas 


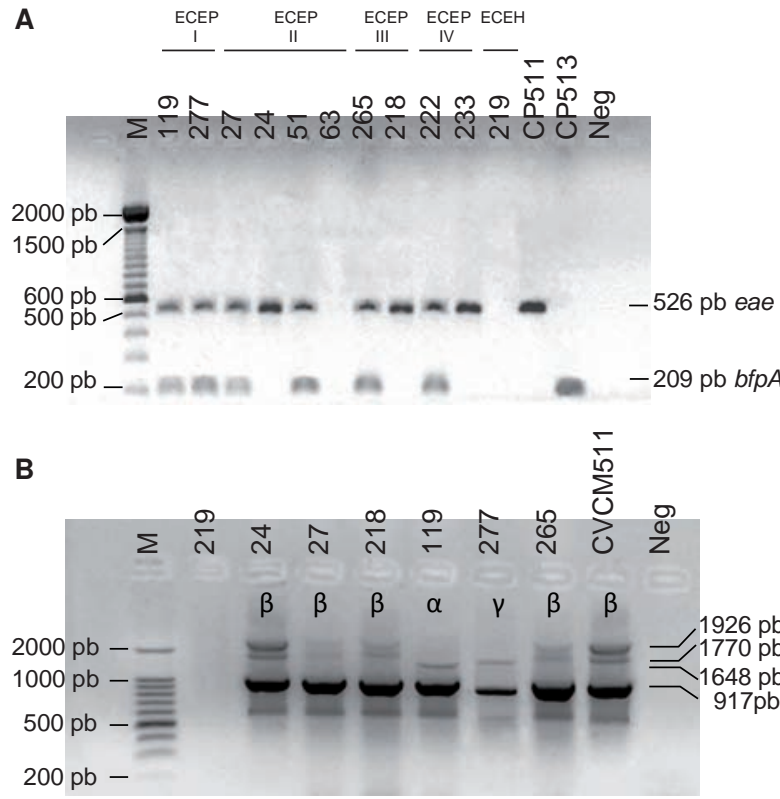

Figura 1. A. Amplificación por PCR múltiple de los genes de intimina (eae) y producción de fimbrias (bfp) con fragmentos de 526 y 209 pb, respectivamente. Electroforesis en gel de agarosa al $2 \%$ teñido con bromuro de etidio. Cepas utilizadas: 119 y 277 (identificadas como ECEP I); 27, 24, 51 y 63 (ECEP II); 265 y 218 (ECEP III); 222 y 233 (ECEP IV); la cepa 219 se identificó como ECEH. Controles positivos (CP): cepas CVCM511 y CVCM513. Control negativo (Neg): agua. M: marcador de peso molecular de 100 pb (Invitrogen). B. Amplificación por PCR múltiple de los genes de intimina (eae) con las variantes alélicas $\alpha$ (1.648 $\mathrm{pb}), \beta(1.926 \mathrm{pb})$ y $\gamma(1.770 \mathrm{pb})$ y el fragmento común para la mayoría de los genes eae de $917 \mathrm{pb}$. Electroforesis en gel de agarosa al $2 \%$ teñido con bromuro de etidio. Cepas utilizadas: 219 (identificada como ECEH); 24 y 27 (ECEP II); 218 y 265 (ECEP III); 119 y 277 (ECEP I). Control positivo (CP): cepa CVCM 511. Control negativo (Neg): agua. M: marcador de peso molecular de $100 \mathrm{pb}$ (Invitrogen)

casos se obtuvieron amplicones para el gen eae, que $58,8 \%$ (30/51) se clasificó como cepas ECEP típicas (eae+ y bfp+) y que el $41,2 \%(21 / 51)$ restante se identificó como cepas ECEP atípicas (eae+ y bfp-). El 95,1\% (48/51) de las amplificaciones del gen eae se encontró en cepas ECEP (ECEP típicas: $60 \%, 15 / 25$ y ECEP atípicas: $40 \%, 10 / 25$ ); las tres cepas restantes (una ECEP típica y dos ECEP atípicas) se clasificaron serológicamente como no aglutinantes. Las cuatro cepas ECEH con el serotipo O157:H7 identificadas mediante antisueros presentaron fermentación de sorbitol, pero no amplificaron en las PCR para la detección de los genes eae y bfpA.

La distribución de los genes amplificados según el serogrupo de $E$. coli reveló que los mayores porcentajes de cepas ECEP típicas: $50 \%$ (15/30), y ECEP atípicas: 47,6 \% (10/21), se encontraron en el serogrupo ECEP II. El reporte de los alelos de intimina mostró que el alelo $\beta$ fue el más frecuentemente identificado entre las cepas positivas para el gen eae $(74,5 \%, 38 / 51)$. En las cepas ECEP I se detectó la presencia de los alelos a $(57,1 \%, 4 / 7), \beta(28,6 \%, 2 / 7)$ y y $(14,3 \%, 1 / 7)$. En las cepas ECEP II y III solo se detectó la presencia del alelo $\beta(86,7 \%, 13 / 15$ y $71,4 \%, 10 / 14$, respectivamente). En 15,7\% (8/51) de las cepas positivas para el gen eae no hubo amplificación de las formas alélicas evaluadas (cuadro 3 ).

\section{Discusión}

Las cepas de E. coli causantes de diarrea se cuentan entre los agentes etiológicos bacterianos más frecuentemente asociados a enfermedades diarreicas agudas, hecho que confirman los hallazgos de la presente investigación, en la cual se identificaron cepas de $E$. coli en $54,7 \%$ de las 192 muestras de heces de los coprocultivos con infección bacteriana, principalmente en los participantes provenientes del municipio urbano de Sucre y en niños de 0 a 4 años de edad. Estos resultados coinciden con reportes previos de estudios en países en desarrollo, según los cuales las tasas de mortalidad y morbilidad infantil por síndrome diarreico agudo comúnmente se asocian a la edad y a la condición social $(3,10,28-30)$. Además, estas enfermedades son más frecuentes durante los primeros dos años de vida, lo cual refleja un patrón que combina el declive de los niveles de anticuerpos maternos, la pérdida de inmunidad activa en el infante y la introducción de alimentos potencialmente contaminados con bacterias fecales $(1,29)$.

Por otro lado, el diagnóstico serológico permitió identificar un elevado porcentaje de cepas positivas para los serogrupos de ECEP, principalmente ECEP II (38,9\%) y ECEP III (18,9\%), mientras que las cepas ECEP I y ECEP IV se identificaron con menor frecuencia (17,3 y 8,2\%, respectivamente); solo se identificaron una cepa ECEI y cuatro con el serotipo ECEH.

Son numerosos los trabajos en los cuales se reporta un rol determinante de las cepas de E. coli causantes de diarrea en infecciones entéricas, lo que demuestra que su correcta identificación es de invaluable ayuda, particularmente en poblaciones con alto riesgo de contraer enfermedades infecciosas $(1,2,29,30)$. Por otra parte, estos resultados son esenciales para cualquier estrategia de control epidemiológico adecuado y para las correspondientes medidas de prevención (1). 
Cuadro 3. Resultados de la amplificación de los genes bfp y eae según la serotipificación de las cepas de Escherichia coli identificadas en las muestras fecales de los niños estudiados

\begin{tabular}{|c|c|c|c|c|c|c|c|}
\hline \multirow[t]{2}{*}{ Serotipo } & \multirow[t]{2}{*}{$\mathbf{n}$} & \multirow{2}{*}{$\begin{array}{l}\text { Detección del } \\
\text { gen eae }\end{array}$} & \multirow{2}{*}{$\begin{array}{l}\text { Detección del } \\
\text { gen bfp }\end{array}$} & \multicolumn{3}{|c|}{ Formas alélicas del gen eae } & \multirow[t]{2}{*}{ P1/P2 } \\
\hline & & & & $\mathbf{a}$ & $\beta$ & $\mathbf{Y}$ & \\
\hline \multirow[t]{6}{*}{$\operatorname{ECEP}^{\Omega} \mid(\mathrm{N}=17)$} & 2 & + & + & + & - & - & + \\
\hline & 1 & + & + & - & + & - & + \\
\hline & 1 & + & + & - & - & + & + \\
\hline & 2 & + & - & + & - & - & + \\
\hline & 1 & + & - & - & + & - & + \\
\hline & 10 & - & - & - & - & - & - \\
\hline \multirow[t]{4}{*}{ ECEP II ( $\mathrm{n}=38)$} & 13 & + & + & - & + & - & + \\
\hline & 2 & + & + & - & - & - & + \\
\hline & 10 & + & - & - & - & - & + \\
\hline & 13 & - & - & - & - & - & - \\
\hline \multirow[t]{5}{*}{ ECEP III ( $n=19)$} & 7 & + & + & - & + & - & + \\
\hline & 2 & + & + & - & - & - & + \\
\hline & 3 & + & - & - & + & - & + \\
\hline & 2 & + & - & - & - & - & + \\
\hline & 5 & - & - & - & - & - & - \\
\hline \multirow[t]{3}{*}{ ECEP IV $(\mathrm{n}=8)$} & 1 & + & + & - & - & - & + \\
\hline & 1 & + & - & - & - & - & + \\
\hline & 6 & - & - & - & - & - & - \\
\hline $\operatorname{ECEH}^{\tilde{\omega}}(n=4)$ & 4 & - & - & - & - & - & - \\
\hline $\operatorname{ECEl}^{\alpha}(n=1)$ & 1 & - & - & - & - & - & - \\
\hline \multirow[t]{3}{*}{ No aglutinante $(n=18)$} & 1 & + & + & - & + & - & + \\
\hline & 2 & + & - & - & + & - & + \\
\hline & 15 & - & - & - & - & - & - \\
\hline Totales & 105 & 51 & 30 & 4 & 38 & 1 & 51 \\
\hline
\end{tabular}

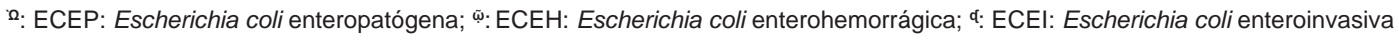

La distribución de los serogrupos de E. coli por edad evidenció que la mayoría se presentó en los niños entre los 0 y 2 años. La característica epidemiológica más sobresaliente de las infecciones por ECEP es su distribución casi exclusivamente en niños menores de dos años; este patrón se ha asociado a los factores socioepidemiológicos determinantes en este grupo de edad y a su inmadurez inmunológica $(10,18,30)$. Asimismo, se ha sugerido que en los mayores de dos años se pueden aislar cepas ECEP sin que haya una correlación estadística entre dichas cepas y los casos de enfermedad infecciosa (31).

Al evaluar los signos y síntomas clínicos de los pacientes, aquellos con resultado positivo para ECEP, además de diarrea acuosa, presentaban moco visible en heces y dolor abdominal. Cabe resaltar que la infección por ECEP puede tener diversos grados de gravedad y que, a diferencia de los resultados de esta investigación, puede presentarse acompañada de vómito y fiebre (16).

Según el serogrupo de E. coli identificado, en los resultados de la amplificación de los genes eae y $b f p A$ predominaron las cepas ECEP típicas frente a las atípicas y en ambos casos el serogrupo ECEP II fue el más frecuente. Este hallazgo es similar al reportado por Blanco, et al. (32), en Uruguay, quienes encontraron una mayor frecuencia de cepas ECEP típicas (57/71) que de ECEP atípicas (14/71) al evaluar 71 aislamientos a partir de muestras fecales de niños con diarrea. Por otra parte, el resultado de la presente investigación difiere de un estudio llevado a cabo en Perú, en el cual se evaluaron niños con diarrea y sin diarrea (grupo de control) y se reportó que las cepas ECEP atípicas fueron el patotipo de E. coli más común en los niños con diarrea (73\%) y en los del grupo de control (87 \%) (33).

Se ha determinado que la distribución geográfica de las cepas ECEP típicas y ECEP atípicas es variable, y que las atípicas predominan en los países industrializados mientras que en los países en desarrollo se observa una baja frecuencia de ECEP típicas y una frecuencia relativamente alta de cepas ECEP atípicas $(34,35)$.

Se ha establecido, asimismo, que las cepas típicas de ECEP están asociadas a la diarrea en niños. Sin embargo, el subgrupo de cepas ECEP 
atípicas se considera como patógeno bacteriano emergente involucrado en casos esporádicos y brotes de diarreas, lo cual exige más estudios epidemiológicos y de virulencia (36).

La falta de correlación entre el diagnóstico serológico y el molecular de estas cepas, es un hecho conocido. En este sentido, se ha señalado una discordancia entre el perfil de patogenia, ya sea por determinación de los genes específicos o de los patrones fenotípicos de adherencia de las cepas, y la pertenencia a los serogrupos de ECEP (37). Sánchez, et al. (35), proponen que la diversidad de cepas circulantes, los eventos de transferencia horizontal, el estado nutricional del huésped, el uso de antibióticos y las condiciones sanitarias, podrían afectar la relación entre los genes de patogenia y virulencia y un serogrupo o serotipo determinado.

El reporte de los alelos de intimina mostró que el $\beta$ fue el más frecuentemente identificado. En los estudios llevados a cabo en este campo se ha evidenciado la acentuada frecuencia con que se identifica el alelo $\beta$ de la intimina en pacientes con cuadros diarreicos y en individuos aparentemente sanos, sin que se observe una correlación entre los serogrupos de E. coli y el tipo de intimina identificado $(15,33,35)$.

La expresión de algunos alelos de la intimina se ha asociado con el tropismo tisular de cepas de E. coli causantes de diarrea (38-41). Sin embargo, la frecuencia de los alelos de intimina evaluados en el presente estudio concordó con otros reportes previos, en los cuales no se encontraron diferencias significativas en la distribución de estos alelos en las cepas ECEP típicas y atípicas, o en la condición clínica de los pacientes $(33,35)$.

En el presente estudio se identificaron ocho cepas positivas para el gen eae en las cuales no hubo amplificación de los alelos de intimina $\alpha, \beta$ y $\gamma$, hecho que podría atribuirse a la gran variabilidad genética descrita para este gen, cuya caracterización ha revelado la existencia de 29 variantes alélicas (32).

Debido a la especificidad de los iniciadores utilizados para los alelos específicos de linaje que codifican Int- $\alpha$, Int- $\beta$ e Int- $\gamma$, este método no solo provee una vía rápida para la identificación de agentes patógenos dentro de los principales grupos clonales de ECEP, sino que constituye una herramienta para su diagnóstico específico en casos en los cuales el tratamiento apropiado es clave en el curso de la enfermedad (42).
La diversidad genética de las cepas de E. coli causantes de diarrea aisladas en personas sanas y en pacientes, se ha comprobado suficientemente, lo que justifica la necesidad de llevar a cabo más estudios epidemiológicos que utilicen herramientas moleculares para detectar factores de virulencia. En el caso particular de las cepas ECEP, esta consideración es clave dado el gran riesgo para la salud pública que representan. Asimismo, este estudio evidenció la importancia del diagnóstico complementario de las pruebas microbiológicas de rutina para la detección e identificación de cepas de E. coli causantes de diarrea, por ser estas las implicadas en condiciones clínicas de diversa gravedad.

\section{Conflicto de intereses}

Los autores declaran no tener conflictos de intereses financieros, políticos o académicos en la elaboración del presente trabajo.

\section{Financiación}

Esta investigación fue parcialmente financiada por el Consejo de Investigación del Núcleo de Sucre de la Universidad de Oriente a través del proyecto identificado con el número Cl-5-1005-1157/03.

\section{Referencias}

1. Organización Mundial de Gastroenterología. Diarrea aguda en adultos y niños: una perspectiva mundial. Guía práctica de la Organización Mundial de Gastroenterología, 2012. Fecha de consulta: 21 de octubre de 2014. Disponible en: http://www.worldgastroenterology.org/assets/.../diarrea_ aguda en adultos.pdf

2. Kaper JB, Nataro JP, Mobley HL. Pathogenic Escherichia coli. Nat Rev Microbiol. 2004;2:123-40. http://dx.doi.org/10. 1038/nrmicro818

3. Onanuga $\mathbf{A}$, Igbeneghu $\mathbf{O}$, Lamikanra $\mathbf{A}$. A study of the prevalence of diarrhoeagenic Escherichia coli in children from Gwagwalada, Federal Capital Territory, Nigeria. J Pan African Med. 2014;17:146. http://dx.doi.org/10.11604/ pamj.2014.17.146.3369

4. Vidal JE, Cañizález-Román A, Gutiérrez-Jiménez J, Navarro-García F. Patogénesis molecular, epidemiología y diagnóstico de Escherichia coli enteropatógena. Salud Pública Mex. 2007:49:376-86.

5. Hernandes RT, De la Cruz M, Yamamoto D, Girón JA, Gomes TA. Dissection of the role of pili and type 2 and 3 secretion systems in adherence and biofilm formation of an atypical enteropathogenic Escherichia coli strain. Infect Immun. 2013;81:3793-802. http://dx.doi.org/10.1128/ IAI.00620-13

6. Nougayrède JP, Fernandes P, Donnenberg MS. Adhesion of enteropathogenic Escherichia coli to host cells. Cell Microbiol. 2003;5:359-72.

7. Nascimento H, Silva L, Souza R, Silva N, Scaletsky I. Phenotypic and genotypic characteristics associated with 
biofilm formation in clinical isolates of atypical enteropathogenic Escherichia coli (aEPEC) strains. BMC Microbiol. 2014;14:184. http://dx.doi.org/10.1186/1471-2180-14-184

8. Nataro J, Kaper J. Diarrheagenic Escherichia coli. Clin Microbiol Rev. 1998; 11:142-201.

9. Zhang WL, Köhler B, Oswald E, Beutin L, Karch H, Morabito S, et al. Genetic diversity of intimin genes of attaching and effacing Escherichia coli strains. J Clin Microbiol. 2002;40:4486-92. http://dx.doi.org/10.1128/JCM. 40.12.4486-4492.2002

10. Moyo SJ, Gro N, Matee MI, Kitundu J, Myrmel H, Mylvaganam $\mathrm{H}$, et al. Age specific aetiological agents of diarrhoea in hospitalized children aged less than five years in Dar es Salaam, Tanzania. BMC Pediatrics. 2011;11:19. http://dx.doi.org/10.1186/1471-2431-11-19

11. Behiry IK, Abada EA, Ahmed EA, Labeeb RS. Enteropathogenic Escherichia coli associated with diarrhea in children in Cairo, Egypt. J Scient World. 2011;11:2613-9. http://dx.doi.org/10.1100/2011/485381

12. Amisano G, Fornasero S, Migliaretti G, Caramello S, Tarasco V, Savino F. Diarrheagenic Escherichia coli in acute gastroenteritis in infants in North-West Italy. New Microbiol. 2011;34:45-51.

13. Paniagua GL, Monroy E, García-González O, Alonso J, Negrete E, Vaca S. Two or more enteropathogens are associated with diarrhoea in Mexican children. Ann Clin Microbiol Antimicrob. 2007;6:17. http://dx.doi.org/10. 1186/1476-0711-6-17

14. de Moura C, Fregolente MC, Martini IJ, Domingos DF, da Silva EJ, Ferraz MM, et al. Prevalence of enteropathogens in normal feces from healthy children at an infant day care in Brazil. J Infect Dev Ctries. 2012;6:176-80. http://dx.doi. org/10.3855/jidc.1982

15. Pitondo-Silva A, Nakazato G, Falcão JP, Irino K, Martínez $\mathbf{R}$, Darini AL, et al. Phenotypic and genetic features of enteropathogenic Escherichia coli isolates from diarrheal children in the Ribeirão Preto metropolitan area, São Paulo State, Brazil. APMIS. 2015;123:128-35. http://dx.doi. org/10.1111/apm.12314

16. Gambandé T, Damiano M, Borda N, Notario R, Aita J. Prevalence of the bacteria causing diarrhea in a Rosario Hospital, Argentina. Rev Fac Cien Med Univ Nac Córdoba. 2006;63:36-8.

17. González R, Díaz C, Mariño M, Cloralt R, Pequeneze M, Pérez-Schael I. Age-specific prevalence of Escherichia coli with localized and aggregative adherence in Venezuelan infants with acute diarrhea. J Clin Microbiol. 1997;35:1103-7.

18. Hannaoui E, Villalobos L, Martínez R, Maldonado A, Hagel I, Bastardo J. Escherichia coli diarreogénica asociada a casos de diarrea aguda en niños de Cumaná, Venezuela. Invest Clin. 2010;51:489-500.

19. Villalobos L, Martínez R, Blanco A, Maldonado A, Bastardo J. Detección molecular de Escherichia coli productor de shiga toxina (Stx1) y rotavirus en heces de niños con diarrea. Invest Clin. 2008;49:387-95.

20. Delannoy S, Beutin L, Fach P. Towards a molecular definition of enterohemorrhagic Escherichia coli (EHEC): Detection of genes located on $\mathrm{O}$ island 57 as markers to distinguish EHEC from closely related enteropathogenic $E$. coli strains. J Clin Microbiol. 2013;51:1083-8. http://dx.doi. org/10.1128/JCM.02864-12

21. Winn WC, Allen SD, Janda WM, Koneman EW, Procop GW, Schrenckenberger PC. Koneman: Diagnóstico microbiológico. Texto y atlas en color. Sexta edición. Buenos Aires: Editorial Médica Panamericana; 2008

22. MacFaddin, J. Pruebas bioquímicas para la identificación de bacterias de importancia clínica. Tercera edición. Buenos Aires: Editorial Médica Panamericana; 2003.

23. Fundación Venezolana para el Estudio de la Salud Infantil (FUVESIN). Productos biológicos. Sueros aglutinantes para el diagnóstico de bacterias productoras de diarrea. Caracas: Universidad Central de Venezuela; 2005.

24. Yamazaki M, Saito $\mathbf{M}$, Inuzaka $\mathbf{K}$, Shima S, Taniwaki $\mathbf{H}$, Itoh K. eaeA genes in Escherichia coli derived from Japanese patients with sporadic diarrea. Kansenshogaku Zasshi. 1997;71:1059-65.

25. Kimata K, Shima T, Shimizu M, Tanaka D, Isobe J, Gyobu Y, et al. Rapid categorization of pathogenic Escherichia coli by multiplex PCR. Microbiol Immunol. 2005;49:485-92. http://dx.doi.org/10.1111/j.1348-0421.2005.tb03752.x

26. Reid S, Betting D, Whittam T. Molecular detection and identification of intimin alleles in pathogenic Escherichia coli by multiplex PCR. J Clin Microbiol. 1999;37:2719-22.

27. Council for International Organizations of Medical Sciences (CIOMS). International ethical guidelines for biomedical research involving human subjects. 2002. Geneva, Switzerland. Fecha de consulta: 21 de octubre de 2014. Disponible en: http://www.cioms.ch/publications/ layout_guide2002.pdf

28. Estrada-García T, López-Saucedo C, Thompson-Bonilla $\mathrm{R}$, Abonce M, López-Hernández $\mathrm{D}$, Santos $\mathrm{Jl}$, et al. Association of diarrheagenic Escherichia coli pathotypes with infection and diarrhea among Mexican children and association of atypical enteropathogenic $E$. coli with acute diarrhea. J Clin Microbiol. 2009;47:93-8. http://dx.doi.org/10. 1128/JCM.01166-08

29. Cabrera-Gaytán DA, Maldonado-Burgos MA, RojasMendoza T, Grajales-Muñiz C. Enfermedad diarreica aguda en niños menores de cinco años de edad: aportaciones de los núcleos trazadores de vigilancia epidemiológica 20122013. Arch Inv Mat Inf. 2013;3:118-25.

30. Orlandi P, Magalhães G, Matos N, Silva B, Penatti M, Nogueira $\mathrm{P}$, et al. Etiology of diarrhea in children of Porto Velho, Amazon region. Braz J Med Biol Res. 2006;39:50717. http://dx.doi.org/10.1590/S0100-879X2006000400011

31. Rappelli P, Folgosa E, Solinas M, Dacosta J, Pisanu C, Sidat M, et al. Pathogenic enteric Escherichia coli in children with and without diarrhea in Maputo, Mozambique. FEMS Immunol Med Microbiol. 2005;43:67-72. http://dx.doi. org/10.1016/j.femsim.2004.07.006

32. Blanco M, Blanco JE, Dahbi G, Mora A, Alonso MP, Varela G, et al. Typing of intimin (eae) genes from enteropathogenic Escherichia coli (EPEC) isolated from children with diarrhoea in Montevideo, Uruguay: Identification of two novel intimin variants (muB and $x i R /$ beta2B). J Med Microbiol. 2006;55:1165-74. http://dx.doi.org/10.1099/jmm. 0.46518-0 
33. Contreras CA, Ochoa TJ, Lacher DW, DebRoy C Navarro A, Talledo M, et al. Allelic variability of critical virulence genes (eae, bfpA and perA) in typical and atypical enteropathogenic Escherichia coli in Peruvian children. J Med Microbiol. 2010;59:2531. http://dx.doi.org/10.1099/ jmm.0.013706-0

34. Trabulsi R, Keller R, Gomes T. Typical and atypical enteropathogenic Escherichia coli. Emerg Infect Dis. 2002; 8:508-13. http://dx.doi.org/10.3201/eid0805.010385

35. Sánchez S, Romecín P, Guachalla L, Iñiguez V. Caracterización geno-fenotípica de aislados de Escherichia coli (AEEC) de pacientes pediátricos con procesos diarreicos infecciosos en la ciudad de La Paz: implicancias para el diagnóstico y epidemiología de las enfermedades diarreicas agudas. Arch Pediatr Urug. 2006;77:195-208. http://dx.doi. org/10.4067/S0370-41062006000400013

36. Magalhães CA, Rossato SS, Barbosa AS, dos Santos TO, Elias WP, Sircili MP, et al. The ability of haemolysins expressed by atypical enteropathogenic Escherichia coli to bind to extracellular matrix components. Mem Inst Oswaldo Cruz. 2011;106:146-52. http://dx.doi.org/10.1590/S007402762011000200005

37. Watterworth L, Topp E, Schraft H, Leung K. Multiplex PCR-DNA probe assay for the detection of pathogenic Escherichia coli. J Microbiol Methods. 2005;60:93-105. http://dx.doi.org/10.1016/j.mimet.2004.08.016
38. Torres AG, Zhou X, Kaper J B. Adherence of diarrheagenic Escherichia coli strains to epithelial cells. Infect Immun. 2005;73:18-29. http://dx.doi.org/10.1128/IAI.73.1.18-29.2005

39. Phillipsa AD, Navabpoura S, Hicksa S, Douganc G, Wallisb T, Frankelc G. Enterohaemorrhagic Escherichia coli 0157:H7 target Peyer's patches in humans and cause attaching/effacing lesions in both human and bovine intestine. Gut. 2000;47:377-81. http://dx.doi.org/10.1136/ gut.47.3.377

40. Cantey JR, Inman LR. Diarrhea due to Escherichia coli strain RDEC-1 in the rabbit: The Peyer's patch as the initial site of attachment and colonization. J Infect Dis. 1981;143:440-6. http://dx.doi.org/10.1093/infdis/143.3.440

41. Fitzhenry RJ, Pickard DJ, Hartland EL, Reece S, Dougan G, Phillips AD, et al. Intimin type influences the site of human intestinal mucosal colonization by enterohaemorrhagic Escherichia coli O157:H7. Gut. 2002;50: 180-5. http://dx.doi.org/10.1136/gut.50.2.180

42. Vidal R, Vidal M, Lagos R, Levine M, Prado V. Multiplex PCR for diagnosis of enteric infections associated with diarrheagenic Escherichia coli. J Clin Microbiol. 2004;42: 1787-9. http://dx.doi.org/10.1128/JCM.42.4.1787-1789.2004 